[Article]

www.whxb.pku.edu.cn

\title{
反相微乳液模板法合成介孔聚苯乙烯
}

\author{
路 霞 唐 静范玉冰胡 军* 刘洪来 \\ (华东理工大学化学系, 结构可控先进功能材料及其制备教育部重点实验室, 上海 200237)
}

\begin{abstract}
摘要：提出并实施了反相微乳液为模板合成介孔聚合物的新方法. 通过考察氯仿/CTAB/水三元系反相微乳 液的稳定性, 以及均相微乳液粒径变化规律, 得到了三元反相微乳液体系的稳定区域. 以 $\left(\mathrm{NH}_{4}\right)_{2} \mathrm{~S}_{2} \mathrm{O}_{8}$ 为引发剂, 利用此反相微乳液为模板合成得到介孔聚苯乙烯材料. 产物的 XRD 图谱中有两个明显的衍射峰, 对应的晶面 间距离 $d$ 分别为 2.6 和 $1.3 \mathrm{~nm}, 1 / d$ 值之比等于 $1: 2$, 为层状结构. $\mathrm{N}_{2}$ 气吸附/脱附曲线表明大部分孔径为 1.7 $\mathrm{nm}$, 与动态光散射测得的微乳液液滴的尺寸相一致.
\end{abstract}

关键词：介孔材料；聚苯乙烯； 反相微乳液；模板；层状结构

中图分类号：0648; O614

\section{Synthesis of Mesoporous Polystyrene Using Reversed Microemulsion Template Method}

\author{
LU Xia TANG Jing FAN Yu-Bing HU Jun* LIU Hong-Lai \\ (Laboratory for Advanced Materials, Department of Chemistry, East China University of Science and Technology, \\ Shanghai 200237, P. R. China)
}

\begin{abstract}
Using a reversed microemulsion as template, we developed a new and convenient synthesis method for mesoporous polymer materials. Based on a study of the stability and sizes of microemulsions at different mass ratios of $\mathrm{CHCl}_{3} / \mathrm{CTAB} / \mathrm{H}_{2} \mathrm{O}$, a stable reversed microemulsion region was discovered. The stable reversed microemulsion was used as a reaction system for the preparation of mesoporous polystyrene. The initiator $\left(\mathrm{NH}_{4}\right)_{2} \mathrm{~S}_{2} \mathrm{O}_{8}$ was dissolved in the reversed microemulsion's water core and polymerization of styrene was initiated at the oil-water interface of the microemulsion. With one intense and another weak diffraction peak, the synthesized polystyrene gave a typical XRD pattern for a lamellar mesostructure. The corresponding lamellar spacing of the (100) and (200) planes were 2.6 and $1.3 \mathrm{~nm}$, respectively. The $\mathrm{N}_{2}$ adsorption/desorption isothermal suggested that the average pore size of the mesoporous polystyrene was mostly $1.7 \mathrm{~nm}$ which coincided with the size of water drops in the reversed microemulsion.
\end{abstract}

Key Words: Mesoporous material; Polystyrene; Reversed microemulsion; Template; Lamellar structure

1992 年, Kreage 等 ${ }^{[1]}$ 成功地合成出包括六方相 MCM-41、立方相 MCM-48 和层状相 MCM-50 等具 有规则孔道的介孔硅基分子篮, 此后介孔材料已 成为国内外学者研究的一个热点 ${ }^{[2,3]}$. 介孔材料具有 孔道规则有序、密度小、孔隙率高、比表面积大等优 ${ }^{\text {点 }}{ }^{[4]}$, 因其显著的表面效应在选择性吸附、形态选择
性催化、分离等领域具有广阔的应用前景 ${ }^{[5,6]}$. 迄今 合成的介孔材料主要为无机材料, 高分子有序介孔 材料的合成尚处于探索阶段. 由于高分子链的柔性 和流动性, 大部分合成体系为非水体系等特征, 合 成具有规则介观结构的高分子材料是一个巨大的 挑战, 无论是合成方法还是材料类型都十分有限.

Received: July 29, 2008; Revised: October 10, 2008; Published on Web: November 11, 2008.

*Corresponding author. Email: junhu@ecust.edu.cn; Tel:+8621-64252921.

国家自然科学基金(20776045, 20736002), 长江学者创新团队(IRT0721), 111 引智计划(B08021)和上海市自然科学基金(07ZR14029)资助项目

C. Editorial office of Acta Physico-Chimica Sinica 
丰富多彩的高分子材料已成为这个世界不可缺少 的部分, 具有纳米或微米孔道的高分子材料兼具高 分子材料本体的特性, 又具有超高的表面效应, 将 在生物反应器、导电材料、传感器以及催化领域成 为一类有着广阔应用前景的新型材料 ${ }^{[7-10]}$.

在无机介孔材料合成中, 软模板技术是重要手 段之一, 通常是以表面活性剂为模板剂, 在水溶液 中与无机前驱体通过界面反应, 形成无机离子聚集 体包覆于模板剂的有序组装体, 通过焙烧或萃取方 式除去模板剂后, 留下无机骨架, 从而获得无机介 孔材料. 通过改变表面活性剂的种类和用量, 可以 形成不同形状和尺寸的胶束模板, 从而实现对孔结 构的调控 ${ }^{[11,12]}$. 而高分子多孔材料通常采用相分离 法 ${ }^{[13]}$ 和硬模板法 ${ }^{[14]}$ 合成得到. 所谓相分离法, 即通 过有机-有机组装中产生的微相分离, 形成具有特 定结构的高分子材料, 通过浸蚀法或溶解嵌段共聚 物(A-B) 中的某一嵌段组分得到一定形貌的孔道结 构. Chang 等 ${ }^{[15]}$ 研究了酚醛树脂、聚环氧乙烷(PEO) 和聚 $\varepsilon$-己酸内脂 $(\mathrm{PCL})$ 三元共混物的相行为和组分 之间氢键作用的关系. 他们发现, 虽然由这三个高 分子组成的三对二元共混物均是完全互溶的, 但由 于三个组分之间氢键作用的差异, 导致该三元共混 物的相图中存在一个闭环区域, 组成处于该区域的 共混物是不混溶的, 可以形成不同的介观分相结 构. 此项研究为后续的高分子介孔材料的研究提供 了一定的理论指导. Hillmyer 等 ${ }^{[16]}$ 制成了一种规则 的环氧树脂和两性嵌段共聚物的纳米混合物, 但由 于两性嵌段共聚物的除去存在困难, 所以没有得到 多孔的高分子材料. Gin 等 ${ }^{[17]}$ 合成出了一种有着规 则孔道结构的介孔有机材料, 但孔结构对热和溶剂 的稳定性较差.

近年来, 合成无机介孔材料所采用的软模板技 术也逐步成功应用于高分子介孔材料的合成, Zhao 等 ${ }^{[18]}$ 采用三嵌段共聚物表面活性剂 P123 或 F127为 模板, 以溶剂挥发诱导自组装方式合成了具有高度 有序的六方相、立方相结构的介孔酚醛树脂, 并通 过高温焙烧, 形成了介孔碳材料. Jang 等 ${ }^{[19]}$ 选用聚醚 表面活性剂为模板剂, 采用反胶束法合成了介孔聚 丙烯腈, 但该材料的孔道有序性较差. Nishiyama 等 ${ }^{[20]}$ 以间苯二酚/甲醛溶液(RF)和三乙氧基正已烷(EOA) 为前驱体, F127 为模板剂, 在酸性条件下, $400{ }^{\circ} \mathrm{C}$ 时 F127 分解, 出现孔道结构, 进一步在 $800{ }^{\circ} \mathrm{C}$ 下直接 碳化合成出了规整的六方结构介孔碳材料 COU-1.
本文在软模板合成技术的基础上，提出了以有 机溶剂/表面活性剂/水三元系形成的反相微乳液为 反应介质, 合成介孔高分子材料的新方法. 采用氯 仿 $\left(\mathrm{CH}_{3} \mathrm{Cl}\right)$, 十六烷基三甲基溴化铵 $(\mathrm{CTAB})$ 和水三 元反相微乳液为反应介质、 $\left(\mathrm{NH}_{4}\right)_{2} \mathrm{~S}_{2} \mathrm{O}_{8}$ 为引发剂、苯 乙烯为聚合物单体, 二乙烯基苯为交联剂成功地合 成了层状介孔聚苯乙烯, 并对反相微乳液法合成介 孔聚合物的机理进行了探讨.

\section{1 实验部分}

\section{1 反相微乳液的研究}

将 $\mathrm{CTAB}$ (分析纯, 上海凌峰化学试剂有限公 司)、 $\mathrm{CHCl}_{3}$ (分析纯, 上海化学试剂有限公司)和去离 子水 (二次重蒸)配制成一系列不同质量比的溶液, $m\left(\mathrm{CHCl}_{3}\right) / m(\mathrm{CTAB}) / m\left(\mathrm{H}_{2} \mathrm{O}\right)=7.5: 1.5: m_{1}\left(m_{1}=0-4\right)$, 超声振荡 $10 \mathrm{~min}$, 混合均匀, 然后分别在 $40{ }^{\circ} \mathrm{C}$ 恒温 $58 \mathrm{~h}$, 考察水对反相微乳液稳定性的影响. 同样配 制质量比 $m\left(\mathrm{CHCl}_{3}\right) / m(\mathrm{CTAB}) / m\left(\mathrm{H}_{2} \mathrm{O}\right)=7.5: m_{2}: 0.3$ $\left(m_{2}=0-3\right)$, 考察 CTAB 对反相微乳液稳定性的影响. 为更系统地考察三元反相微乳液的稳定性, 固定 $\mathrm{CHCl}_{3}$ 的量, 在油包水反相微乳液范围内随机加人 水和表面活性剂, 配制成系列不同质量比的溶液.

\section{2 粒径测定}

采用英国马尔文公司 Nano-ZS 型动态光散射 仪在 $40{ }^{\circ} \mathrm{C}$ 下测定溶液中反相微乳液的粒径, 激光 源 $\mathrm{He}-\mathrm{Ne}, 4.0 \mathrm{mV}, 633 \mathrm{~nm}$, 动态光散射-非侵人式 背散射技术.

\section{3 介孔聚苯乙烯的合成}

将过硫酸铵 $\left(\mathrm{NH}_{4}\right)_{2} \mathrm{~S}_{2} \mathrm{O}_{8}$ (分析纯, 上海凌峰化学 试剂有限公司)溶于水中, 并与适量比例的 CTAB 和 $\mathrm{CHCl}_{3}$ 混溶, 超声混合 $10 \mathrm{~min}$, 然后在 $40{ }^{\circ} \mathrm{C}$ 下恒温 搅拌 $2 \mathrm{~h}$. 将苯乙烯和二乙烯基苯加人反应体系中, 继续摚拌 $2 \mathrm{~min}$ 后停止搅拌, 静置. 反应进行若干 时间后, 得到白色聚苯乙烯样品. 将上述聚苯乙烯 样品 $1 \mathrm{~g}$, 加人乙醇 $100 \mathrm{~mL}$, 盐酸 $18 \mathrm{~mL}, 70{ }^{\circ} \mathrm{C}$ 回流 $8 \mathrm{~h}$, 提取模板剂. 然后抽滤, 常温真空干燥 $24 \mathrm{~h}$.

\section{4 介孔苯乙烯的表征}

XRD 测试在日本理学 D/Max2550 VB/PC 型 $X$ 射线粉末衍射仪上进行, 管电压 $40 \mathrm{kV}$, 管电流 $200 \mathrm{~mA}$, 铜靶, 小角扫描区间为 $1^{\circ}-10^{\circ}$; 吸附/脱附 等温线在 Micrometrics ASAP 2020 型物理吸附仪 上测量, 吸附质为 $\mathrm{N}_{2}$, 温度为 $-196{ }^{\circ} \mathrm{C}$, 样品的孔径 分布由 BJH 法处理得到. 


\section{2 结果与讨论}

\section{$2.1 \mathrm{CHCl}_{3} / \mathrm{CTAB} / \mathrm{H}_{2} \mathrm{O}$ 反相微乳液}

$\mathrm{CHCl}_{3} / \mathrm{CTAB} / \mathrm{H}_{2} \mathrm{O}$ 三元系统在适当的配比下可 形成均一稳定的微乳液. 本文采用的高分子单体能 溶于 $\mathrm{CHCl}_{3}$, 为了合成有序的具有介观结构的聚合 物材料, 必须选择 $\mathrm{CHCl}_{3}$ 为连续相的油包水型反向 微乳液系统, 即 CTAB 反胶束中包裹了微量的水. $40{ }^{\circ} \mathrm{C}$ 下, 氯仿、水和表面活性剂的三元局部相图如 图 1 所示. 图中坐标单位均为质量分数, 曲线所围 内部为均相区. $1_{1}$ 线为保持氯仿和水的质量比不变, 考察表面活性剂量的变化对系统稳定性的影响. 当 CTAB 的含量比较少时, 形成的反胶束还不足以容 纳所有的水, 因此游离在胶束外的水和氯仿形成互 不相溶的两相. 随着 CTAB 量增加, 形成的反胶束 量增多, 当足以包裹全部水时, 反相微乳液系统为 均相. 保持氯仿和 CTAB 质量比不变, 考察水量变 化的影响, 如 $1_{2}$ 线, 随水含量的上升, 系统由均相变 为非均相. 合成聚合物介孔材料所需的反相微乳液 反应体系处在均相区域内.

反相微乳液的物理性质主要取决其含水量 $W_{0}$,

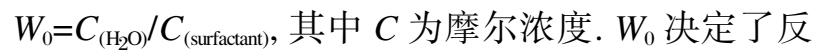
相微乳液滴的大小和每个液滴中所含表面活性剂 的数目. 图 2 是反相微乳液粒径随含水量 $W_{0}$ 的变 化情况.

当 $W_{0}$ 较小时, 少量的水被包裹在反相微乳液 的内核中, 微乳液粒径基本不变, 为 $1.7 \mathrm{~nm}$ 左右. $W_{0}$ 大于 7.0 , 反相微乳液液滴随水含量的增加, 粒 径有显著的增加. 可以预计, 随着 $W_{0}$ 继续增加, 反 相微乳液系统将失去稳定性.

\section{2 介孔聚苯乙烯的表征}

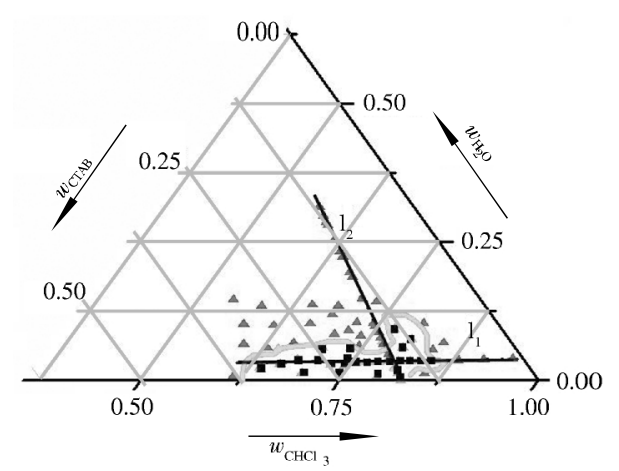

图 $140{ }^{\circ} \mathrm{C}$ 时, $\mathrm{CHCl}_{3} / \mathrm{CTAB} / \mathrm{H}_{2} \mathrm{O}$ 系统三元相图

Fig.1 Ternary phase diagram of the $\mathrm{CHCl}_{3} / \mathrm{CTAB} /$ $\mathrm{H}_{2} \mathrm{O}$ system at $40{ }^{\circ} \mathrm{C}$

represents the homogeneous phase, and $\boldsymbol{\Delta}$ represents the heterogeneous phase.

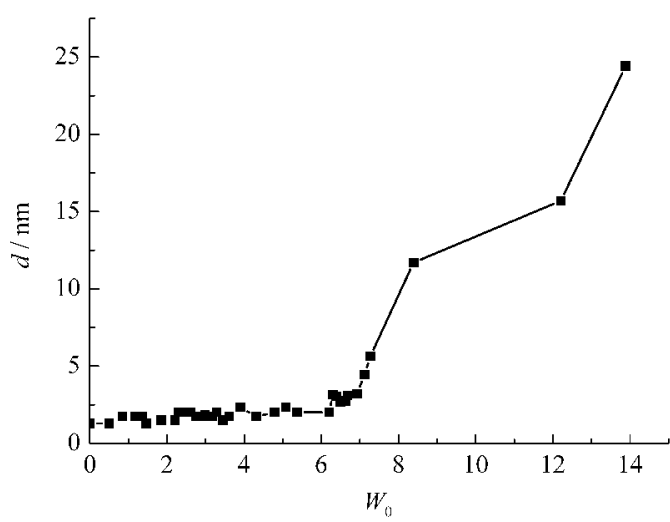

图 2 反相微乳液粒径 $(d)$ 随含水量 $\boldsymbol{W}_{0}$ 的变化

Fig.2 Size $(d)$ changes of reversed microemulsion with $\boldsymbol{W}_{0}$

$W_{0}=C_{\left(\mathrm{H}_{2} \mathrm{O}\right.} / C_{\text {(surfactant) }}$

选取上述 $W_{0}<7.0$ 的稳定微乳液体系, 通过微 乳液诱导、自由基聚合得到了白色粉末状聚苯乙烯. 其 XRD 图谱如图 3 所示, 在衍射角 $2 \theta=3.3^{\circ}$ 时出现 明显的衍射峰 1 , 表明其具有一定的介观结构. 同 时在 $2 \theta=6.7^{\circ}$ 处还有一弱衍射峰 2 , 根据布拉格公 式 $2 d \sin \theta=n \lambda(n=1)$ (式中 $d$ 为晶面间距离, $\theta$ 为衍射 角, $n$ 为衍射级数, $\lambda$ 为 $\mathrm{X}$ 射线波长), 衍射峰对应 的晶面间距离 $d$ 分别为 2.6 和 $1.3 \mathrm{~nm}$, 相应衍射晶 面分别为(100)、(200), $1 / d$ 值的比为 $1: 2$, 为典型的层 状相 ${ }^{[21]}$. 由此可以判定合成产物聚苯乙烯具有层状 介观结构. 此外, 衍射峰 1 前的微小峰为 CTAB 特 征峰, 表明还有微量 CTAB 没有除尽.

层状结构的聚苯乙烯对 $\mathrm{N}_{2}$ 气的吸附/脱附曲 线如图 4(a)所示, 为典型的 II 型吸附/脱附线, 吸附 量在较低相对压力 $\left(p / p_{0}\right)$ 时迅速增加, 在中间相对 压力时, 吸附量继续升高, 但变化不明显, 几乎平行 于相对压力坐标轴, 表明孔径较小, 在相对压力趋

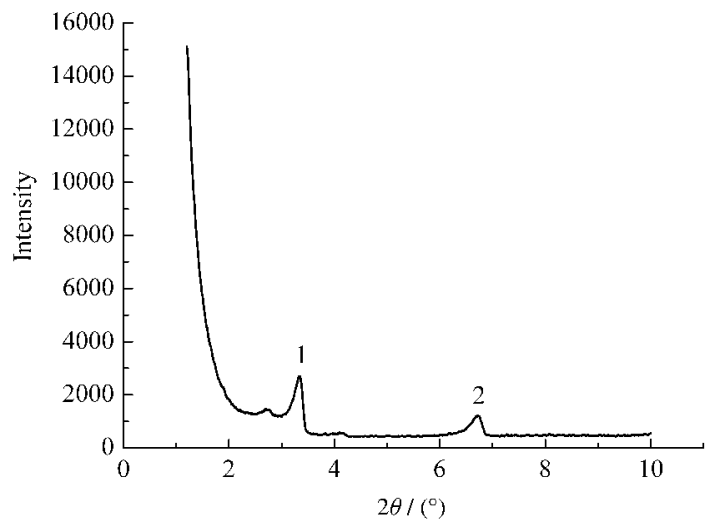

图 3 聚苯乙烯的 XRD 图

Fig.3 XRD pattern of PS mesoporous material 

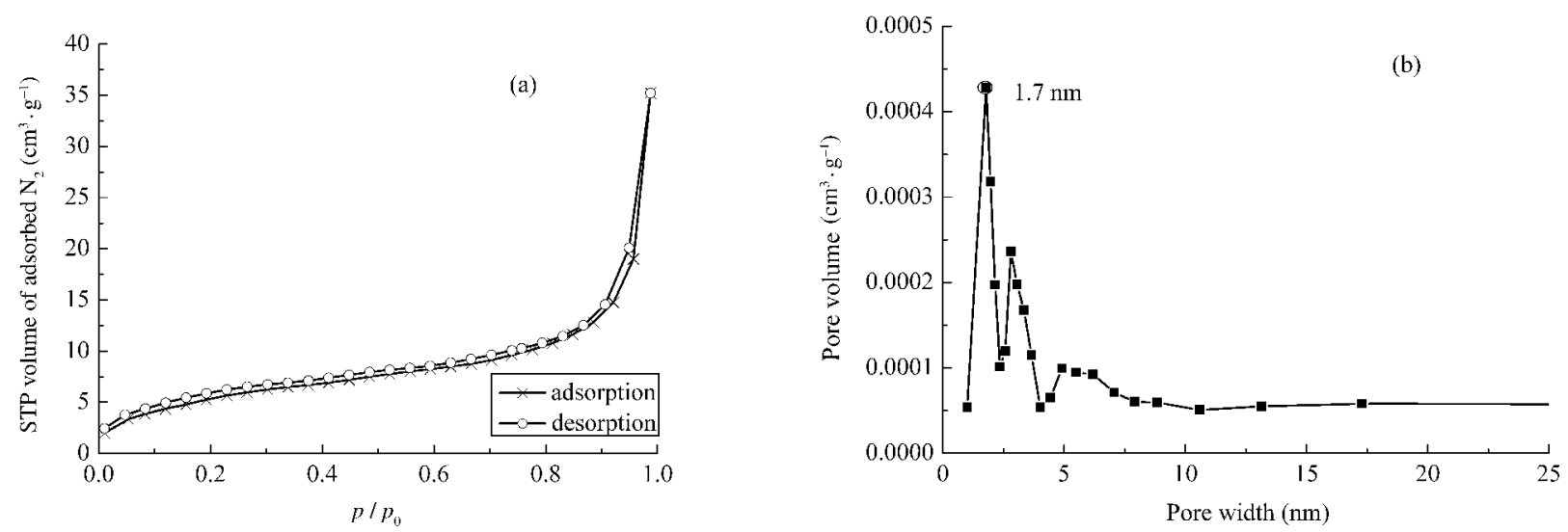

图 4 聚苯乙烯的 $(a) \mathbf{N}_{2}$ 气吸附/脱附曲线和(b)采用 $\mathbf{B J H}$ 模型处理脱附曲线得到的孔径分布曲线

Fig.4 (a) Adsorption and desorption isotherm of $\mathrm{N}_{2}$ on PS mesopourous material, (b) the pore size distribution curve by the BJH analysis of desorption branch isotherm

近于 1 时, 吸附量增加, 没有出现吸附极限, 可能是 材料外表面吸附所致.氮气的总体吸附量较小, 表 明材料的比表面积较小. 这可能有两种原因引起, 其一, 由于聚合物链具有一定的柔性, 模板剂提取 后致使聚合物层状结构坞塌; 其二, 由于聚合物链 具有一定的流动性, 模板剂提取过程中溶剂的热回 流致使部分孔道口被游离的聚合物链堵塞. 部分结 构的坍塌和孔道堵塞致使 $\mathrm{N}_{2}$ 气吸附分析中, 气体 无法进人内孔道, 氮气的总体吸附量较小. 通过BJH 模型对脱附曲线进行计算, 得到的孔径分布如图 4 (b), 平均孔径为 $7 \mathrm{~nm}$, 其中大部分孔径为 $1.7 \mathrm{~nm}$, 与上述微乳液液滴的尺寸相匹配. 进一步证明聚苯
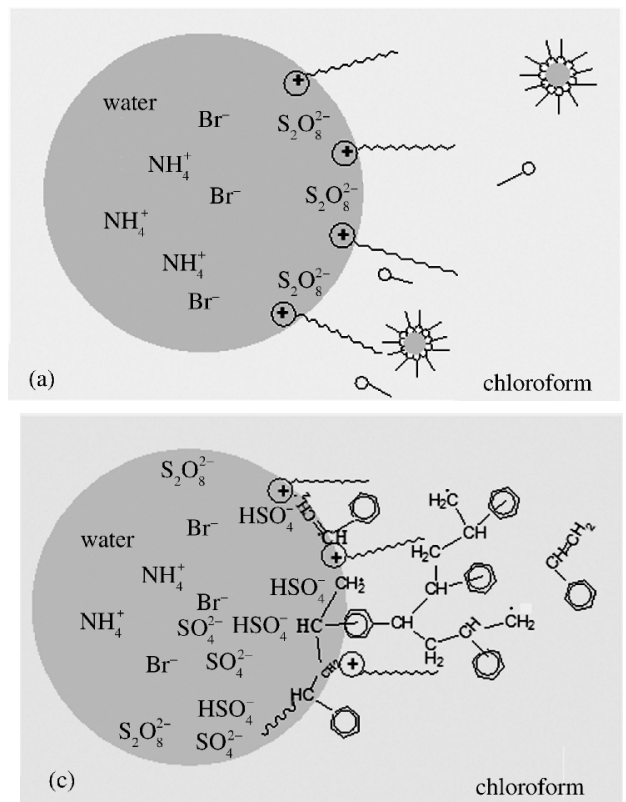

$\mathrm{H}_{2} \mathrm{O}$
乙烯的层状结构是由反向微乳液模板诱导形成的.

\section{3 反相微乳液模板法合成介孔聚苯乙烯的机理}

实验结果表明利用反相微乳液合成介孔聚合 物的设计方法具有可行性, 为使反相微乳液法成为 简单而通用的合成介孔聚合物的新方法, 探讨其合 成机理是极为必要的. 图 5 给出了采用 $\mathrm{CHCl}_{3} /$ $\mathrm{CTAB} / \mathrm{H}_{2} \mathrm{O}$ 反相微乳液系统合成介孔聚苯乙烯的 机理. 包含四个步骤: 微乳液形成 $\rightarrow$ 界面引发 $\rightarrow$ 聚 合 $\rightarrow$ 除模板.

如图 5(a)所示, 在一定配比下 $\mathrm{CHCl}_{3} / \mathrm{CTAB} / \mathrm{H}_{2} \mathrm{O}$ 三元系统形成反相微乳液滴, CTAB 头基聚集在水 相, 尾链伸展于 $\mathrm{CHCl}_{3}$ 油相中. 水溶性引发剂过硫酸
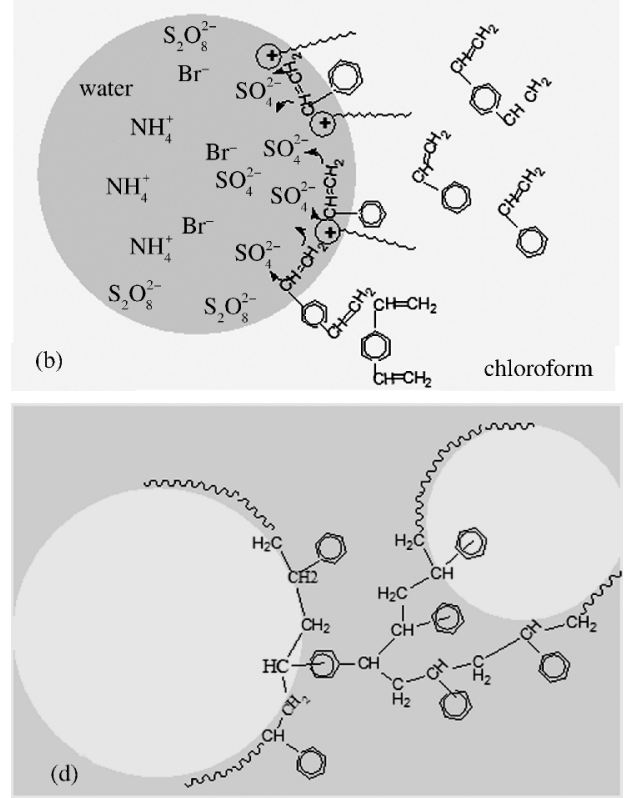

Oil

图 5 由反向微乳液合成介孔聚苯乙烯的机理图

Fig.5 Schematic procedure of synthesis of mesoporous PS by the reversed microemulsion template 
铵 $\left(\mathrm{NH}_{4}\right)_{2} \mathrm{~S}_{2} \mathrm{O}_{8}$ 被 CTAB 包裏在水滴内核, 相互碰撞发 生反应形成自由基. 聚合物单体苯乙烯和二乙烯基 苯溶解在 $\mathrm{CHCl}_{3}$ 油相中, 在反相微乳液 CTAB-油相 界面的苯乙烯和二乙烯基苯单体捕获到 $\left(\mathrm{NH}_{4}\right)_{2} \mathrm{~S}_{2} \mathrm{O}_{8}$ 分解产生的自由基, 从而引发自由基聚合反应, 如 图 5(b)所示. 自由基既可能沿着微乳液表面传递, 在界面生成聚合物; 也可能逐步向油相传递, 在油 相生成连续的聚合物; 其中二乙烯基苯起交联剂作 用, 使油相中的聚合物形成网状结构, 从而保留并 固定微乳液模板剂的结构, 如图 5(c)所示. 聚合反应 结束后, 通过乙醇破乳, 并除去表面活性剂模板, 即 得到聚合物母体中含有规则孔道的介孔聚合物材 料, 如图 5(d).

介孔聚合物材料的介孔结构决定于模板剂的 结构, 但单体在聚合过程中有可能通过挤压、聚集等 相互作用方式, 改变软模板的结构形貌, 最终获得 的聚合物介孔结构与初始的模板结构不一定完全 相同. 聚合过程对介孔结构的影响有待进一步研究.

\section{3 结 论}

在合适的比例下 $\mathrm{CHCl}_{3} / \mathrm{CTAB} / \mathrm{H}_{2} \mathrm{O}$ 三元系可 以形成均一的反相微乳液. 在含水量 $W_{0}<7.0$ 时, 此 反相微乳液稳定, 液滴粒径为 $1.7 \mathrm{~nm}$ 左右基本不 变. 继续增加 $W_{0}$, 反相微乳液系统失去稳定性. 以 稳定的均一反相微乳液为模板, 水溶性 $\left(\mathrm{NH}_{4}\right)_{2} \mathrm{~S}_{2} \mathrm{O}_{8}$ 为引发剂, 通过界面引发、体相聚合形成了聚苯乙烯 材料, 产物 XRD 图有两个明显的衍射峰, 其 $1 / d$ 值 比为 $1: 2$, 表明具有层状结构, 其(100)面层间距离 为 $2.6 \mathrm{~nm} . \mathrm{N}_{2}$ 气吸附/脱附曲线表明大部分孔道直 径为 $1.7 \mathrm{~nm}$. 以反相微乳液为模板合成介孔聚合物 的机理包含四个步骤: 微乳液形成 $\rightarrow$ 界面引发 $\rightarrow$ 聚合 $\rightarrow$ 除模板, 该机理为简便合成介孔聚合物提供 了新思路.

\section{References}

1 Kreage, C. T.; Leonowicz, M. E.; Roth, W. J.; Vartuli, J. C.; Beck, J. S. Nature, 1992, 359: 710

2 Impens, N. R. E. N.; van der Voort, P.; Vansant, E. F. Micropor. Mesopor. Mater., 1999, 28: 217

3 Zhang, Z. R.; Suo, J. S.; Zhang, X. M.; Li, S. B. Progress in Chemistry, 1999, 11(1): 11 [张兆荣, 索继栓, 张小明, 李树本. 化学进展, 1999, 11(1): 11]

4 Li, Y.; Kim, S. J. J. Phys. Chem. B, 2005, 109: 12309

5 Ryoo, R.; Joo, S. H.; Yun, S. J. Phys. Chem. B, 1999, 103: 7743

6 Attard, G. S.; Bartlett, P. N.; Coleman, N. R. B.; Elliott, J. M.; Owen, J. R.; Wang, J. H. Science, 1997, 278: 2373

7 Green, P. F.; Limary, R. Adv. Colloid Interface, 2001, 94: 53

8 Peters, E.; Svec, C. F.; J. Frechet, M. J. Adv. Mater., 1999, 11: 1169

9 Hedrick, J. L.; Miller, R. D.; Hawker, C. J.; Carter, K. R.; Volksen, W.; Yoon, D. Y.; Trollsas, M. Adv. Mater., 1998, 10: 1049

10 de Boer, B.; Stalmach, U.; Nijland, H.; Hadziioannou, G. Adv. Mater, 2000, 12: 1581

11 Fajula, F.; Galarneau, A.; Renzo, F. D. Micropor. Mesopor. Mater. 2005, 82: 227

12 Hu, J.; Zhou, L. H.; Li, H. N.; Li, W. X.; Liu, H. L.; Hu, Y. Acto Phys. -Chim. Sin., 2005, 21(11): 1217 [胡＼cjkstart军, 周丽绘, 李鸿宁, 李万全金, 刘洪来, 胡 英. 物理化学学报, 2005, 21(11): 1217]

13 Jenekhe, S. A.; Chen, X. L. Science, 1999, 283: 372

14 Johnson, S. A.; Ollivier, P. J.; Mallouk, T. E. Science, 1999, 283 963

15 Kuo, S. W.; Lin, C. L.; Chang, F. C. Macromolecules, 2002, 35 : 278

16 Lipic, P. M.; Bates, F. S.; Hillmyer, M. A. J. Am. Chem. Soc., 1998, 120: 8963

17 Miller, S. A.; Kim, E.; Gray, D. H.; Gin, D. L. Angew. Chem. Int. $E d .$, 1999, 38(20): 3021

18 Meng, Y.; Gu, D.; Zhang, F. Q.; Shi, Y. F.; Yang, H. F.; Li, Z.; Yu, C. Z.; Tu, B.; Zhao, D. Y. Angew. Chem. Int. Ed., 2005, 44: 7053

19 Jang, J.; Bae, J. Chem. Commun., 2005, (9): 1200

20 Tanaka, S.; Nishiyama, N.; Egashira, Y.; Ueyama, K. Chem. Commun., 2005, (16): 2125

21 Ma, L. D. Modern X-ray powder diffraction. Beijing: Chemical Industrial Press, 2004: 16-90 [马礼敦. 近代 X 射线多晶体衍 射. 北京: 化学工业出版社, 2004: 16-90] 